\title{
Epidemiología de la Leptospirosis en el departamento del Huila, Colombia. 2011- 2017
}

Gustavo Cedeño Chacón a1; Juan Manuel Gómez Cerquera ${ }^{\text {b1 }}$; María Valentina Chica Polanía c1; Angie Vanessa Arce Polo ${ }^{\text {d1 }}$; William Perdomo Flórez ${ }^{\mathrm{e}}$; Yaliana Tafurt Cardona ${ }^{\mathrm{f} 1}$.

\begin{abstract}
${ }^{a}$ Médico general. Joven Investigador Colciencias. ORCID: https://orcid.org/0000-0003-4101-2422, gustavo.cedeno@ uninavarra.edu.co ; b Médico internista. ORCID: https://orcid.org/0000-0001-7278-8296, juanmac90@hotmail.com ; ' Médica general. ORCID: https://orcid.org/0000-0002-7508-2835, valenchica19@gmail.com ; ${ }^{\mathrm{d}}$ Médica general. ORCID: https://orcid.org/0000-0002-0661-5589, vanesaapolo@ hotmail.com ; ${ }^{\mathrm{e}}$ Médico veterinario y zootecnista.

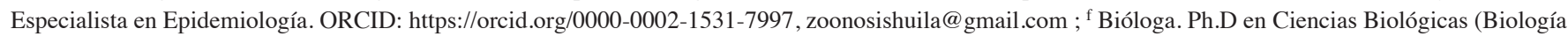
Celular y Molecular). ORCID: https://orcid.org/0000-0003-1289-2395, yalianat@gmail.com

${ }^{1}$ Fundación Universitaria Navarra-UNINAVARRA. Facultad de Ciencias de la Salud. Neiva, Colombia ${ }^{2}$ Secretaría de Salud Departamental del Huila. Vigilancia Epidemiológica de Zoonosis. Salud Pública. Neiva, Colombia. DOI $10.22517 / 25395203.24549$
\end{abstract}

\section{Resumen}

Objetivo: Describir la epidemiología de la Leptospirosis en el departamento del Huila durante el período 2011 $-2017$.

Materiales y métodos: Estudio descriptivo de corte transversal, retrospectivo. La población analizada fueron pacientes de los diferentes municipios del departamento del Huila que se reportaron como casos probables o confirmados de leptospirosis. Se estructuraron registros por cada año en bases de datos recopiladas por el área de epidemiología de la Secretaría de Salud del Huila, basadas en las fichas de notificación de vigilancia en salud pública para leptospirosis y generadas por cada Unidad Primaria Generadora de Datos (UPGD) ante la presencia del evento. Los datos fueron discriminados de acuerdo a las variables de interés para el estudio en una nueva base de datos en Excel; tabuladas con el programa estadístico R - Studio 3.3.3 y Epidat 4.2, para luego ser procesados con estadísticos descriptivos y finalmente ser expresados en porcentajes y en tasas de incidencia.

Resultados: Se reportaron 268 casos, de los cuales 61 (23\%) fueron confirmados por laboratorio y 207 (77\%) sospechosos o probables. El $69 \%$ de los pacientes presentaron fiebre, mialgias y cefalea como síntomas representativos. El grupo etario más afectado tenía entre 27 y 59 años de edad (44\%). La población se caracterizó por ser del área urbana $(65 \%)$, con mayor frecuencia en hombres $(72 \%)$. En relación a la ocupación laboral, se encontró mayor frecuencia en agricultores (20\%) y en el personal de aseo (17\%). Los factores de riesgo evidenciados fueron la presencia de animales domésticos en un $67 \%$ de los casos, así como la presencia de ratas en el hogar (51\%) y el posible mal manejo del agua.
Conclusiones: El incremento en el número de casos de Leptospirosis probablemente está asociado a la mala manipulación del agua y a la presencia de animales en el domicilio.

Palabras clave: Leptospirosis, Epidemiología, Colombia.

\section{Epidemiology of leptospirosis in the department of Huila. 2011 to 2017}

\begin{abstract}
Objective: Describe the epidemiology of Leptospirosis in the department of Huila during the years 2011 to 2017.
\end{abstract}

Materials and methods: This article is a descriptive, cross-sectional, retrospective study. The population analyzed were patients from the different municipalities of the department of Huila who were reported as probable or confirmed cases for leptospirosis, structuring records for each year in databases compiled by the epidemiology area of the department's health secretary, based on the public health surveillance notification sheets for leptospirosis, and generated by each Primary Data Generating Unit (UPGD) in the presence of the event; The data were discriminated according to the variables of interest for the study in a new database in Excel; tabulated with the statistical program R - Studio 3.3.3 and Epidat 4.2 , to later be processed with descriptive statistics and finally expressed in percentages and incidence rates.

Results: 268 cases were reported, which 61 (23\%) were confirmed by laboratory and 207 (77\%) suspicious or probable. $69 \%$ of the patients presented fever, myalgia and headache as representative symptoms. The most affected group range in ages from 27 and 59 (44\%). 
The population was characterized for being part of the urban area (65\%), and the cases were more frequent in men $(72 \%)$. Regarding to employment, it was found that farmers $(20 \%)$ and the cleaning staff $(17 \%)$ were the most common cases. The risk factors identified were the presence of domestic animals in $67 \%$ of the cases, as well as the presence of rats at home (51\%) and the possible poor water management.

Conclusions: The increase in the number of cases of Leptospirosis is probably associated with the poor water management and the presence of animals at home.

Key words: Leptospirosis, Epidemiology, Colombia

\section{Introducción}

La Leptospirosis es una enfermedad infecciosa de orden mundial que se trasmite de animales a humanos, considerándose como una zoonosis con un alto potencial epidémico, principalmente en las regiones tropicales (1). La principal forma de transmisión se produce por el contacto directo de un humano con la orina de roedores, perros, vacas, cerdos y animales silvestres que estén infectados, además de aguas o ambientes contaminados (2). El patógeno responsable de la enfermedad es el Leptospira interrogans es del cual se han descrito más de 200 especies que pueden infectar al hombre (3). Según la Organización Mundial de la salud (OMS) la leptospirosis puede, potencialmente, constituir una enfermedad grave pero puede ser tratada dado que su cuadro clínico es similar a otras infecciones no relacionadas, tales como influenza, meningitis, hepatitis, dengue o fiebres virales hemorrágicas; si no es tratada a tiempo, puede ser mortal (4). De acuerdo con la Organización Panamericana de la Salud (OPS), se estima que a nivel mundial cada año se registran 1,03 millones de casos de leptospirosis, causando aproximadamente 58.900 muertes (5).

En la Región de las Américas, la tasa anual de morbilidad fluctúa entre un mínimo de 3,9 casos por cada 100.000 habitantes con mayor incidencia en el Sur de América Latina y un máximo de 50,7 casos en el Caribe (5). Durante el año 2014 fueron notificados 10.702 casos humanos a nivel mundial, de los cuales el $95,5 \%$ se registró en América Latina, con un mayor número de casos en Brasil (3.974), Perú (2.329) y Colombia (867). En el Caribe, los índices más altos se presentaron en Trinidad y Tobago con 363 casos, San Vicente y las Granadinas con 17 casos y República Dominica con 10 casos (5).
En Colombia, la prevalencia de la enfermedad varía de acuerdo a la región, por ejemplo, en una comunidad indígena del departamento de Córdoba, un estudio demostró que la seroprevalencia fue de 18,1\%; en la región de Urabá, Antioquia, la prevalencia fue de 12,5\%; confirmando así, la circulación de Leptospira spp en diversas regiones del país (6) Según, Carreño et al, en Colombia, principalmente en la Costa Atlántica, Urabá antioqueño y el Eje Cafetero, se presentan los índices más altos de la enfermedad (7).

El departamento del Huila, ha evidenciado una curva creciente de casos notificados, encontrando 73 casos durante el año 2013, mientras que en los años 2014 y 2015 se reportaron 95 y 85 casos respectivamente. Para el año 2016 se evidenció un incremento significativo en el número de casos para un total de 118 , aunque para el año 2017 la cifra disminuyó a 46 casos notificados (8).

En este contexto, el Plan Decenal de Salud Pública (PDSP) Colombia 2012 - 2021 y el Ministerio de Salud y Protección Social diseñaron, en el año 2015, la Estrategia de Gestión Integrada de las zoonosis para el $100 \%$ de las Direcciones Territoriales de Salud, con el objetivo de apoyar la prevención de la Leptospirosis. Con ello, se espera que para el año 2021, se conozca de manera global y por departamentos, la tasa de incidencia de esta infección en todo Colombia (9), así como estudios de vigilancia epidemiológica contemplados en el PDSP, los cuales son requeridos para establecer los determinantes de la infección y su frecuencia, con el fin de conocer el impacto real de la enfermedad y su importancia en entornos rurales o urbanos del país (10).

Para lograr un mejor conocimiento de la enfermedad, es importante contar con estudios epidemiológicos tanto locales como nacionales que permitan conocer con más detalle el comportamiento de esta patología, facilitándoles a las entidades pertinentes diseñar estrategias de salud pública en intervención y prevención. Teniendo en cuenta lo anterior, el objetivo de este estudio fue describir la epidemiologia de la Leptospirosis en el departamento del Huila durante el período 2011- 2017. 


\section{Materiales y métodos:}

Los casos registrados fueron tomados de las bases de datos del departamento de vigilancia epidemiológica de la secretaria de salud Departamental del Huila, que fueron reportados como casos probables y confirmados por laboratorio durante el período 2011-2017.

Se realizó un estudio descriptivo, retrospectivo de corte transversal. La base de datos incluía información sociodemográfica, datos clínicos, nexo epidemiológico y resultados de laboratorio (IgM contra Leptospira en muestras pareadas mediante microaglutinación (MAT), cultivo de Leptospira spp en cualquier fluido corporal o por detección del ADN mediante Reacción en Cadena de la Polimerasa); no hubo discriminación por edad, sexo, ni raza.

El procesamiento y el análisis de datos se realizó con el programa estadístico $\mathrm{R}$ Comander. Las variables continuas se expresaron en media \pm desviación estándar, las variables discretas se expresaron en frecuencias y proporciones. Posteriormente, se realizó un análisis estadístico mediante la prueba de chi-cuadrado (x2) para la comparación de proporciones y la tasa de incidencia estandarizada de cada uno de los municipios por cada 100.000 habitantes y se graficó en un mapa del departamento del Huila.

\section{Resultados}

Un total de 268 casos de Leptospirosis fueron reportados durante el período de estudio. La edad promedio de los pacientes fue de 34,11 + 19,24. El 44\% de la población estudio eran adultos, el $72 \%$ eran masculinos y en su mayoría provenientes del área urbana (65\%); las actividades laborales asociadas con riesgo para la infección, presentes en el 39\% de los casos, fueron la agricultura y personal que labora en actividades de aseo. Los síntomas más frecuentes fueron fiebre, cefalea o mialgias, encontrados en un $69 \%$ de los casos. Los datos se registran en la Tabla 1. 
Epidemiología de la Leptospirosis en el departamento del Huila, Colombia. 2011- 2017 Rev. Méd Risaralda 2021;vol 27 No.1

\begin{tabular}{|c|c|c|}
\hline \multicolumn{2}{|r|}{ CARACTERÍSTICAS } & $\begin{array}{c}\text { POBLACIÓN } \\
\text { N (\%) }\end{array}$ \\
\hline \multicolumn{2}{|l|}{ TOTAL } & $268(100 \%)$ \\
\hline \multirow{7}{*}{$\begin{array}{c}\text { Edad/ } \\
\text { grupo etarios }\end{array}$} & MEDIA \pm DS & $34,11 \pm 19,24$ \\
\hline & Primera Infancia & $10(4)$ \\
\hline & Infancia & $13(5)$ \\
\hline & Adolescencia & $51(19)$ \\
\hline & Juventud & $44(16)$ \\
\hline & Adultez & $118(44)$ \\
\hline & Adulto Mayor & $32(12)$ \\
\hline \multirow{2}{*}{ Género } & Masculino & $194(72)$ \\
\hline & Femenino & $74(28)$ \\
\hline \multirow{2}{*}{ Lugar de residencia } & Urbana & $175(65)$ \\
\hline & Rural & $93(35)$ \\
\hline \multirow{2}{*}{ Ocupación } & Ocupación con Riesgo para la Infección & $105(39)$ \\
\hline & Ocupación sin Riesgo para la Infección & $163(61)$ \\
\hline \multirow{4}{*}{ Síntomas } & Asintomático & $0(0)$ \\
\hline & $\begin{array}{l}\text { Síntomas constitucionales (fiebre, mialgias o } \\
\text { cefalea) }\end{array}$ & $186(69)$ \\
\hline & $\begin{array}{l}\begin{array}{l}\text { Síntomas específicos (esplenomegalia o } \\
\text { ictericia) }\end{array} \\
\end{array}$ & $10(4)$ \\
\hline & 2 o más & $72(27)$ \\
\hline
\end{tabular}

Tabla 1. Caracterización sociodemográfica de la población estudio.

El municipio con mayor tasa de incidencia de casos notificados fue Elías con 7 casos por cada 100.000 habitantes, seguido de La Plata, con 6 casos por cada 100.000 habitantes (Figura

Elías y La Plata fueron los 2 municipios del departamento del Huila con la tasa más alta de incidencia: 7 y 6 casos por cada 100.000 habitantes respectivamente. 

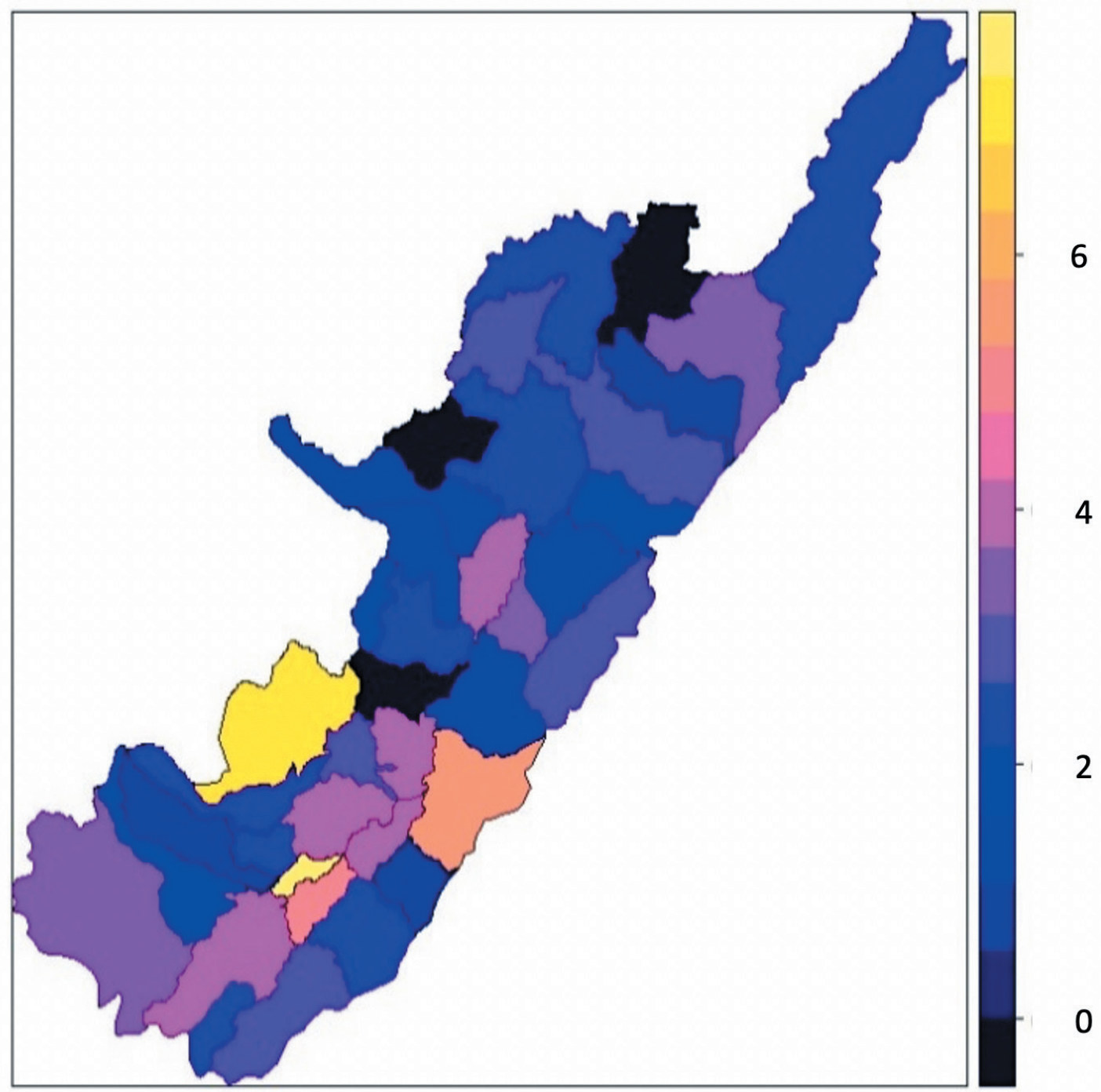

Figura 1. Tasas de incidencia por 100.000 habitantes, discriminado por municipios según los casos notificados durante el período 2011 - 2017.

Como se indica en la Tabla 2, la población de estudio fue discriminada de acuerdo a las definiciones operativas de los tipos de casos que se describen en el protocolo de vigilancia en salud pública para la Leptospirosis. De los 268 pacientes, 61 fueron casos confirmados por laboratorio (23\%) y 207 (77\%) identificados como probables o sospechosos. Los años con mayor número de casos reportados fueron el 2014 y 2015 con un total de 56 casos para el año 2014 (21\% del total de casos del período en estudio) y de 50 casos para el año 2015 (19\% del total de casos del período en estudio). 


\begin{tabular}{|c|c|c|c|}
\hline Características & $\begin{array}{r}\text { Caso probable o sos- } \\
\text { pechoso } \\
\mathrm{N}(\%) \\
\end{array}$ & $\begin{array}{c}\text { Caso confirmado por } \\
\text { laboratorio } \\
\mathbf{N}(\%) \\
\end{array}$ & $P$ \\
\hline Total & $207(77)$ & $61(23)$ & N.A \\
\hline \multicolumn{4}{|l|}{ Año de notificación } \\
\hline 2011 & $12(4)$ & $5(2)$ & \\
\hline 2012 & $28(10)$ & $16(6)$ & \\
\hline 2013 & $29(11)$ & $2(1)$ & \\
\hline 2014 & $42(16)$ & $14(5)$ & \\
\hline 2015 & $37(14)$ & $13(5)$ & \\
\hline 2016 & $42(16)$ & $3(1)$ & \\
\hline 2017 & $17(6)$ & $8(3)$ & $0,006^{\mathrm{a}}$ \\
\hline \multicolumn{4}{|l|}{ Fuentes de agua } \\
\hline No reportado & $8(3)$ & $1(0,5)$ & \\
\hline Acueducto & $150(56)$ & $47(17,5)$ & \\
\hline Río & $19(7)$ & $6(2)$ & \\
\hline Pozo comunitario & $17(6,5)$ & $6(2)$ & \\
\hline $\begin{array}{l}\text { Tanque de } \\
\text { almacenamiento }\end{array}$ & $6(2)$ & $1(0,5)$ & \\
\hline $\begin{array}{l}\text { Más de } 2 \text { fuentes de } \\
\text { agua }\end{array}$ & $7(3)$ & o (o) & $0,647^{\mathrm{a}}$ \\
\hline \multicolumn{4}{|l|}{ Presencia de ratas } \\
\hline Sí & $106(39)$ & $32(12)$ & \\
\hline No & $101(38)$ & $29(11)$ & $0,864^{\mathrm{a}}$ \\
\hline \multicolumn{4}{|l|}{ Alcantarillas destapadas } \\
\hline Sí & $59(22)$ & $11(4)$ & \\
\hline No & $148(55)$ & $50(19)$ & $0,102^{a}$ \\
\hline \multicolumn{4}{|l|}{ Presencia de aves de corral } \\
\hline Sí & $10(4)$ & $2(1)$ & \\
\hline No & $197(73)$ & $59(22)$ & $0,606^{a}$ \\
\hline \multicolumn{4}{|l|}{ Presencia de bovinos y porcinos } \\
\hline Sí & $25(9)$ & $6(2)$ & \\
\hline No & $182(68)$ & $55(21)$ & $0,631^{\mathrm{a}}$ \\
\hline \multicolumn{4}{|l|}{ Presencia de animales domésticos } \\
\hline Sí & $134(50)$ & $46(17)$ & \\
\hline No & $73(27)$ & $15(6)$ & $0,119^{a}$ \\
\hline \multicolumn{4}{|l|}{ Contacto con animales enfermos } \\
\hline $\mathrm{Si}$ & $18(7)$ & $5(2)$ & \\
\hline No & $189(70)$ & $56(21)$ & $0,903^{a}$ \\
\hline \multicolumn{4}{|l|}{ Inundaciones en zona de vivienda } \\
\hline Sí & $12(5)$ & $4(1)$ & \\
\hline No & $195(73)$ & $57(21)$ & $0,826^{\mathrm{a}}$ \\
\hline \multicolumn{4}{|l|}{ Contacto con aguas estancadas } \\
\hline Sí & $45(17)$ & $12(5)$ & \\
\hline No & $162(60)$ & $49(18)$ & $0,729^{a}$ \\
\hline
\end{tabular}




\begin{tabular}{|l|c|c|c|}
\hline Disposición de residuos solidos & & & \\
\hline Recolección & $134(50)$ & $38(14)$ & \\
\hline $\begin{array}{c}\text { Disposición } \\
\text { Peridomiciliaria }\end{array}$ & $73(27)$ & $23(9)$ & $0,727^{\text {a }}$ \\
\hline Tiempo de almacenamiento de basura & & & \\
\hline $1-3$ Días & $142(53)$ & $41(15)$ & \\
\hline $4-7$ Días & $60(23)$ & $17(6)$ & \\
\hline Más de 7 días & $5(2)$ & $3(1)$ & $0,600^{\text {a }}$ \\
\hline Antecedentes de actividades deportivas & & & \\
\hline Sí & $70(26)$ & $19(7)$ & \\
\hline No & $137(51)$ & $42(16)$ & $0,697^{\text {a }}$ \\
\hline
\end{tabular}

DS = Desviación estándar, $P$ : probabilidad

Tabla 2. Distribución de la población según el tipo de caso notificado en relación a los factores de riesgos asociados a la infección por Leptospirosis.

Uno de los principales hallazgos se centra en las fuentes de agua para las actividades del hogar ya que en el $9 \%$ de los casos notificados se evidenció la utilización de agua de los ríos para la ingesta y quehaceres del hogar. En cuanto a la presencia de animales domésticos en el hogar se encontró que el $26 \%$ de los casos estuvo expuesto a este factor de riesgo. Por otro lado, la presencia de ratas en el hogar o en el lugar de trabajo se identificó en el $51 \%$ de los pacientes.

Situaciones predisponentes descritas clásicamente en la literatura, como la exposición a fuentes de aguas estancadas y presencia peridomiciliaria de alcantarillas destapadas, no fueron identificadas en la población estudiada. Sin embargo, las condiciones socio-sanitarias si tuvieron relevancia en los casos reportados, ya que el $24 \%$ de los pacientes confirmados con Leptospirosis tenían residuos sólidos (basura) a nivel domiciliario y peridomiciliario; con respecto a las ocupaciones laborales, solo el 39\% de los pacientes tenían un oficio que se asocia con el riesgo para adquirir la enfermedad. (Trabajos en agricultura, ganadería, veterinarios, pescadores, personal de aseo, del ejército y policías).

\section{Discusión}

Generalmente, las zoonosis pasan desapercibidas, ya sea porque los propios animales no desarrollan la enfermedad o porque los síntomas comienzan a manifestarse en los seres humanos después de un largo período de tiempo (12). Los casos de leptospirosis registrados durante los años de estudio, en el departamento del Huila, nos indican que existe una transmisión directa o indirecta del agente etiológico de los animales al hombre a través del contacto con ambientes contaminados (13)the brown rat (Rattus norvegicus. Según Macías Herrera y colaboradores, la leptospirosis se puede presentar en todas las etapas de la vida, alcanzando mayores tasas de incidencia en edades de vida económicamente productiva, siendo coherente con este estudio, donde el mayor número de casos se presentaron en adultos (14). Esta investigación mostró a la agricultura como la ocupación más predominante en los casos registrados. Sethi y colaboradores, indican que la vida agraria es un factor de riesgo debido al íntimo contacto con animales y campos inundados, los cuales son condiciones adecuadas para la supervivencia y transmisión de la leptospira (15) Según el sistema de información regional del Huila, la cosecha de arroz es la actividad agropecuaria más frecuente (16); un estudio realizado en agricultores dedicados al cultivo de arroz en Perú, reveló que la práctica de trabajar descalzo y la manipulación de roedores en los campos de cultivo, predispone a la enfermedad (17).

Diferentes estudios han mostrado que la prevalencia de la leptospirosis es mayor en hombres que en mujeres y se encuentra directamente relacionado a los roles desempeñados en labores productivas agropecuarias, 
siendo más susceptibles al riesgo de la enfermedad; lo anterior coincide con datos reportados en este estudio, donde el sexo masculino tiene mayor porcentaje de casos confirmados (18). Un dato a destacar en esta investigación es el hecho de tener el mayor número de casos en el área urbana, lo cual puede estar asociado a que la agricultura urbana surge como un proceso de expansión territorial que sirve como mecanismo para albergar familias rurales que llegan a las ciudades buscando una mejor calidad de vida (19). Dicha migración promueve las prácticas agropecuarias en los espacios urbanos y periurbanos y genera modificaciones en los ecosistemas aumentando la presencia de enfermedades como la leptospira(15).

La expansión de los barrios marginales urbanos ha creado condiciones propicias para la transmisión de la infección por ratas (20). Según la literatura científica, los roedores son los principales reservorios de Leptospira spp, ya que se desplazan libremente y pueden tener contacto con otras especies de animales facilitando la diseminación de la infección para hospederos susceptibles; el diagnóstico en estos animales es difícil y su infección no induce síntomas lo que dificulta aún más el control de estos reservorios (21). En este estudio, se evidenció la presencia de ratas y animales domésticos en lugares de trabajo o vivienda, lo cual predispone a la infección.

Cabe recordar que los reservorios naturales entre los animales domésticos incluyen el ganado bovino, los cerdos, las ovejas y los perros, los cuales varían con la serovariedad y la región geográfica (22). En las zonas rurales, el ganado bovino vive en estrecho contacto con los trabajadores agrícolas; se ha detectado una prevalencia relativamente alta de leptospirosis en la orina de bovinos, convirtiéndose en una importante vía de transmisión a través de la orina contaminada (13)the brown rat (Rattus norvegicus.

Las condiciones geográficas, climatológicas, económicas y sociales de Colombia, generan un ambiente propicio para la reemergencia de la leptospirosis; su ubicación tropical, con pisos térmicos cálidos en la mayoría del territorio nacional, condiciones sociales no adecuadas, trabajo sin protección laboral, servicios sanitarios deficientes y asentamientos poblacionales, favorecen la aparición de la enfermedad (23).
Por otro lado, es importante señalar que diversas investigaciones han sugerido una asociación entre los cambios climáticos y la presentación de una mayor cantidad de casos de la enfermedad (24). Un estudio realizado en Tailandia, reporta mayor número de casos después de la temporada de lluvias e inundaciones en áreas tropicales (25).

En la presente investigación, los años que reportaron mayor número de casos de leptospirosis fueron el 2011 y 2016. Según el IDEAM los cambios climáticos reportados en esos años surgieron drásticamente como consecuencia del fenómeno de "La niña" en algunas regiones de Colombia, entre ellas el departamento del Huila, potenciando este factor de riesgo para la transmisión de la Leptospira (26). En este estudio se identificó que los municipios que presentaron mayor tasa de incidencia fueron Elías y La Plata, en donde la actividad laboral está centrada principalmente en actividades agrícolas y pecuarias (27)(28). Según el Análisis Situacional en Salud (ASIS), estos municipios presentan determinantes sociales de la salud precarios, deficiencia en la cobertura del alcantarillado, del acueducto, en la calidad del agua y condiciones sanitarias desfavorables, haciendo que enfermedades como la Leptospirosis puedan afectar más fácilmente a esta población (28)(27).

La frecuencia de los casos notificados a nivel mundial es variable y depende de las características sociales y climatológicas de la población, además no se conoce precisamente el número de casos humanos, debido el subdiagnóstico o diagnóstico erróneo de la enfermedad (3). Estudios nacionales e internacionales, indican que la presencia de las manifestaciones clínicas como fiebre, dolor de cabeza, mialgia, malestar general, dolor abdominal, diarrea y artralgia hacen parte de la sintomatología inicial de la leptospirosis pero que a la vez crea confusión por la similitud de los síntomas generados por otros microorganismos (29); estas investigaciones son concordantes con lo hallado en este estudio, ya que los síntomas que más se evidenciaron en los casos notificados fueron la fiebre, cefalea y mialgias.

Finalmente, debido a que la sintomatología es variable y que el cuadro clínico es similar a otras enfermedades endémicas del departamento del Huila, como el Dengue, el diagnóstico de Leptospirosis no es fácil y requiere 
de una alta sospecha clínica y epidemiológica para detectarlos a tiempo. Es importante un control de los vectores (control de roedores, ganado) y una mejora de las condiciones socio-sanitarias por parte de las entidades competentes para reducir el número de casos de la enfermedad y mediante un diagnóstico oportuno evitar el impacto en la calidad de vida de la población.

\section{Agradecimientos:}

Secretaría de Salud departamental del Huila

\section{Referencias}

1. Caino H, Scagalia J, Crucio F, Siquiroff G. Leptospirosis. Rev Fac Ciencias médicas. 2006;1(3):30-6.

2. Rodríguez R. Evaluación del comportamiento de la leptospirosis humana mediante un modelo matemático atendiendo a variables climáticas como predictoras. [Internet] 2010 [citado el 19 de diciembre de 2018]. Disponible en: http://www.veterinaria.org/ revistas/redvet/n030310B/0310B DS27.pdf

3. Organización Mundial de la Salud. Leptospirosis. [Internet] 2021[citado el 15 de diciembre 2020]. Disponible en: https://www.paho.org/

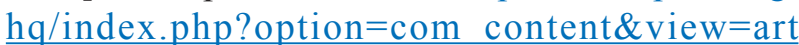
icle\&id=7821:2012-informacion-general-leptospirosis\&Itemid $=0 \&$ lang $=\mathrm{es}$

4. Terpstra WJ. Leptospirosis Humana: Guía Para el Diagnóstico, Vigilancia y Control [Internet] 2008 [citado el 19 de diciembre de 2019]. Disponible en: http://www.med.monash.edu.au/microbiology/staff/ adler/guia-esp.pdf

5. World Health Organization. The control of neglected zoonotic diseases : From advocacy to action: Report of the fourth international meeting held at WHO headquarters, Geneva, Switzerland, 19-20 November 2014. [Internet] 2014 [citado el 19 de diciembre de 2019]. Disponible en: https://www.paho.org/ salud-en-las-americas-2017/?post t es=zoonosis

6. Flores P. Leptospirosis humana en Colombia: la experiencia del Instituto Colombiano de Medicina Tropical - CES. CES Med [Internet] 2008 [citado el 19 de diciembre de 2019];21(1). Disponible en: http://revistas.ces.edu.co/index.php/medicina/article/view/118

7. Buitrago L. Informe nacional de zoonosis 2014. [Internet] 2014 [citado el 15 de diciembre de 2019]. Disponible en: https://www.slideshare.net/LuisAlbertoCarreoBui/informe-nacional-de-zoonosis-2014

8. Secretaría de Salud Depatamental del Huila. Boletines Epidemiológicos año 2017 [Internet] 2017 [citado el 19 de diciembre de 2019] Disponible en: https:// www.huila.gov.co/salud/publicaciones/7191/boletines-epidemiologicos-ano-2017/

9. Ministerio de Salud y Protección Social. Plan Decenal de Salud Pública Colombia 2012 - 2021. Sistema de seguimiento. [Internet] 2021 [citado el 10 de enero de 2021]. Disponible en: https://www.minsalud. gov.co/Documentos y Publicaciones/Sistema de Seguimiento y Evaluación del Plan Decenal De Salud Pública - PDSP Colombia 2012 - 2021.pdf

10. Ministerio de Salud y Protección Social. Plan decenal de salud publica 2012 - 2021 [Internet] 2013 [citado el 19 de diciembre de 2019]. Disponible en: https://www.minsalud.gov.co/Documentos y Publicaciones/Plan Decenal - Documento en consulta para aprobación.pdf

11. Gobernación del Huila. Análisis de Situación en Salud del Departamento del Huila - Neiva 2016 [Internet] 2016 [citado el 19 de diciembre de 2019]. Disponible en: http:/huila.gov.co/documentos/2017/ saludpublica/ASIS/asis departamental-2016-huila. pdf

12. Katz AR, Ansdell VE, Effler P V, Middleton CR, Sasaki DM. Leptospirosis in Hawaii, 1974-1998: epidemiologic analysis of 353 laboratory-confirmed cases. Am J Trop Med Hyg [Internet] 2002 [cited 2018 Dec 19];66(1):61-70. Disponible en: http:// www.ncbi.nlm.nih.gov/pubmed/12135270

13. Haake DA, Levett PN. Leptospirosis in Humans. In: Current topics in microbiology and immunology [Internet] 2015 [cited 2018 Dec 19]. p. 65-97. Disponible en: http://www.ncbi.nlm.nih.gov/pub$\mathrm{med} / 25388133$

14. Agudelo-Flórez P, Restrepo-Jaramillo BN, Arboleda-Naranjo M. Situación de la leptospirosis en el Urabá antioqueño colombiano: estudio seroepide- 
miológico y factores de riesgo en población general urbana. Cad Saude Publica [Internet] 2007 [citado el 19 de diciembre de 2019];23(9):2094-102.Disponible en: http://www.scielo.br/scielo.php?script=sci arttext\&pid=S0102-311X2007000900017\&lng=es \&tlng=es

15. Sethi S, Sharma N, Kakkar N, Taneja J, Chatterjee SS, Banga SS, et al. Increasing trends of leptospirosis in northern India: a clinico-epidemiological study. Martins EAL, editor. PLoS Negl Trop Dis [Internet]2010 [cited 2018 Dec 19];4(1):e579. Available from: https://dx.plos.org/10.1371/journal. pntd.0000579

16. Gobernación del Huila. Economía [Internet] 2017 [citado el 19 de diciembre de 2019]. Disponible en: https://www.huila.gov.co/publicaciones/148/econo$\underline{\mathrm{mia} /}$

17. Tejada Caminiti R, Romaní Romaní F, Wong P, Alarcón Villaverde J. Prácticas laborales de riesgo en cultivadores de arroz del valle del Alto Mayo, Región San Martín, Perú. Rev Peru Epidemiol [Internet] 2011 [citado el 19 de diciembre de 2019];15(1):7. Disponible en: https://dialnet.unirioja.es/servlet/ articulo? codigo $=3750053$

18. CentroProvincial de Información de Ciencias Médicas, Cuba. Caracterización clinicoepidemiológica de pacientes con leptospirosis. MEDISAN [Internet] 2009 [ citado el 19 de diciembre de 2019];13(1). Disponible en: http://scielo.sld.cu/scielo.php?script=sci_artt ext\&pid=S1029-30192009000100004

19. Lacerda HG, Monteiro GR, Oliveira CCG, Suassuna FB, Queiroz JW, Barbosa JDA, et al. Leptospirosis in a subsistence farming community in Brazil. Trans R Soc Trop Med Hyg [Internet] 2008 [cited 2018 Dec 19];102(12):1233-8. Available from: https://academic.oup.com/trstmh/article-lookup/ doi/10.1016/j.trstmh.2008.05.010

20. Costa F, Ribeiro GS, Felzemburgh RDM, Santos $\mathrm{N}$, Reis RB, Santos AC, et al. Influence of household rat infestation on leptospira transmission in the urban slum environment. Small PLC, editor. PLoS Negl Trop Dis [Internet] 2014 [cited 2018 Dec 19];8(12):e3338. Available from: http://dx.plos. org/10.1371/journal.pntd.0003338
21. Mori M, Bourhy P, Le Guyader M, Van Esbroeck M, Djelouadji Z, Septfons A, et al. Pet rodents as possible risk for leptospirosis, Belgium and France, 2009 to 2016. Euro Surveill [Internet] 2017 [cited 2018 Dec 19];22(43). Available from: http://www. eurosurveillance.org/content/10.2807/1560-7917. ES.2017.22.43.16-00792

22. Acha PN SB. Zoonoses And Communicable Diseases Common To Man And Animals [Internet] 2003 [cited 2018 Dec 19].Available from: https://www. paho.org/hq/dmdocuments/2012/Acha-ZoonosesEng.pdf

23. D. Esobar, W. Garcia, L.A Sandoval, L.E. Tibaquira HG. Epidemiologia de la Leptospirosis en el Departamento del Tolima, Colombia, 2009 - 2011. Rev Fac Nac Salud Pública [Internet] 2013 [citado el 19 de diciembre de 2019];31(1):48-57. Disponible en: http://www.scielo.org.co/scielo.php?script=sci arttext\&pid=S0120-386X2013000100006

24. Petrakovsky J, Bianchi A, Fisun H, Nájera-Aguilar P, Pereira MM. Animal leptospirosis in Latin America and the Caribbean countries: reported outbreaks and literature review (2002-2014). Int J Environ Res Public Health [Internet]2014 [cited 2018 Dec 19];11(10):10770-89. Available from: http://www. mdpi.com/1660-4601/11/10/10770.

25. Kawaguchi L, Sengkeopraseuth B, Tsuyuoka R, Koizumi N, Akashi H, Vongphrachanh P, et al. Seroprevalence of leptospirosis and risk factor analysis in flood-prone rural areas in Lao PDR. Am J Trop Med Hyg [Internet] $2008 \mathrm{~J}$ [cited $2018 \mathrm{Dec}$ 19];78(6):957-61. Available from: http://www.ncbi. nlm.nih.gov/pubmed/18541776

26. Instituto de Hidrología, Meteorología y Estudios Ambientales. IDEAM. Tiempo y Clima. [Internet] 2014 [cited 2018 Dec 19]. Available from: http:// www.ideam.gov.co/web/tiempo-y-clima

27. Gobernación del Huila. Análisis de Situación en Salud del Municipio de La Plata, Huila 2017. [Internet] 2017 [cited 2018 Dec 19]. Available from:https:// www.huila.gov.co/salud/loader.php?1Servicio=To ols $2 \& 1$ Tipo $=$ descargas $\& 1$ Funcion $=$ descargar $\&$ idFi le $=26450$ 
28. Gobernacion del Huila. Análisis de Situación en Salud del Municipio de Elías, Huila 2017 [Internet] 2017 [cited 2018 Dec 19]. Available from:https:// www.huila.gov.co/salud/loader.php?1Servicio=To ols2\&1Tipo $=$ descargas $\& 1$ Funcion $=$ descargar\&idFi $1 \mathrm{e}=26442$

29. Arroyave E, Londoño AF, Quintero JC, Agudelo-Flórez P, Arboleda M, Díaz FJ, et al. Etiology and epidemiological characterization of non-malarial febrile syndrome in three municipalities of Urabá (Antioquia), Colombia. Biomédica [Internet] 2013 [citado el 19 de diciembre de 2019];33,1:99-107. Disponible en:http://www.ncbi.nlm.nih.gov/pubmed/24652254 